\title{
dspace.vutbr.cz
}

\section{Design of Fractional-Order Integrator Controlled by Single Voltage Gain}

\author{
ŠOTNER, R.; PETRŽELA, J.; JEŘÁBEK, J.; HERENCSÁR, N.; ANDRIUKAITIS, D.
}

Proceedings of the 2019 42nd International Conference on Telecommunications and Signal Processing (TSP), pp. 360-364

elSBN: 978-1-7281-1864-2

DOI: http://dx.doi.org/10.1109/TSP.2019.8768814

Accepted manuscript

(C2019 IEEE. Personal use of this material is permitted. Permission from IEEE must be obtained for all other uses, in any current or future media, including reprinting/republishing this material for advertising or promotional purposes, creating new collective works, for resale or redistribution to servers or lists, or reuse of any copyrighted component of this work in other works. ŠOTNER, R.; PETRŽELA, J.; JEŘÁBEK, J.; HERENCSÁR, N.; ANDRIUKAITIS, D., "Design of Fractional-Order Integrator Controlled by Single Voltage Gain", Proceedings of the 2019 42nd International Conference on Telecommunications and Signal Processing (TSP), pp. 360-364, 2019. DOl: 10.1109/TSP.2019.8768814. Final version is available at 


\title{
Design of Fractional-Order Integrator Controlled by Single Voltage Gain
}

\author{
Roman Sotner*, Jiri Petrzela*, Jan Jerabek* ${ }^{*}$ Norbert Herencsar ${ }^{*}$, and Darius Andriukaitis $\dagger$ \\ ${ }^{*}$ SIX Research Center, Faculty of Electrical Engineering and Communication, Brno University of Technology, Brno, Czech \\ Republic \\ $\dagger$ Department of Electronics Engineering, Faculty of Electrical and Electronics Engineering, Kaunas University of Technology, \\ Kaunas, Lithuania \\ Email: sotner@feec.vutbr.cz
}

\begin{abstract}
This work presents analyses of interaction of fractional- and integer-order transfer functions when their responses are added together by simple linear operation of sum. The mathematical background is very complex but practical consequences may be very useful for further design of electronically reconfigurable circuits including modification of shape of the resulting magnitude and phase responses. Described approach allows control of constant phase shift in limited operational bandwidth by single parameter that is documented by PSpice simulations with models of common of-the-shelf active elements.
\end{abstract}

Keywords-Constant phase element; fractional-order; integer-order; linear operation; phase shift control; transfer response.

\section{INTRODUCTION}

Systems including or employing fractional-order [1] components start to be important in common technical practice (see for example design of oscillators [2], [3], filters [4], [5], organic impedance modeling [6], regulation and control [7][9], etc.). Many aspects of combination or interconnection of fractional-order elements (two-terminals) has been studied recently [10]-[11]. These works deal with ideal or approximated representation of behavior of fractional-order passive element or the observations are based on real fabricated fractional-order devices constructed in several ways (chemicals/liquids [12], polymer composites [13], ferroelectric materials [11], carbon materials [14], deposited layers of different materials) [15], etc.). These devices are also known as so-called constant phase elements (CPEs) [16], [17].

In recent years, these fractional-order elements were studied in many linear passive and active filtering circuits (for example [4], [5] and references cited therein) or particular impedance generating topologies (for example [6], [18]-[20]) and their electronically controllable applications (see for example integrators and differentiators in [21], [22]). However, the basic mathematical operations of symbolical voltage transfer functions (where one of them has fractional-order character) that are added together have not been studied in detail in past. There are exceptions regarding fractional-order proportional, integral and/or derivative controllers (see for example [6]-[9]) where fractional-order elements (and devices)

This article is based upon work from COST Action CA15225, a network supported by COST (European Cooperation in Science and Technology). Research described in this paper was financed by the National Sustainability Program under grant LO1401 and by the Ministry of Education, Youth and Sports under grant LTC18022 of Inter-Cost program. For the research, infrastructure of the SIX Center was used. are subparts of the system blocks that also contribute to the overall transfer function. However, these controllers are more complex than solution introduced in this paper and require also additional signal path(s). These controllers do not consider other possible utilization of resulting responses and their theoretical suability for different applications. The symbolical expressions for linear mathematical operations in case of systems including fractional-order device are quite complex but the particular settings of circuit features (loop gains, time constants, orders, etc.) may bring interesting features in frequency responses.

This paper brings brief study of resulting transfer response gained as sum of fractional- and integer-order lossy low-pass transfers and some experiments with parameters forming both responses and also overall behavior. The specific setting of the circuit model allows us to obtain variable value of constant phase shift (controlled by single parameter) in limited operational bandwidth.

\section{STUdied Voltage-Mode TOPOLOGY}

The interconnection of fractional-order and integer-order low-pass voltage-mode transfer blocks (lossy integrators) is shown in Fig. 1(a). This concept, redrawn to specific circuit topology (Fig. 1(b)), has the following transfer function:

$$
K_{C}(s)=H(s)+G(s),
$$

where partial transfers $H(s)$ and $G(s)$ have respective forms:

$$
\begin{aligned}
& H(s)=\frac{A}{1+\tau_{1} s^{\alpha}}, \\
& G(s)=\frac{B}{1+\tau_{2} s} .
\end{aligned}
$$

Each low-pass block has own time constant and pass-band gain. The overall transfer function of the system is:

$$
K_{C}(s)=\frac{A}{1+\tau_{1} s^{\alpha}}+\frac{B}{1+\tau_{2} s}=\frac{A\left(1+\tau_{2} s\right)+B\left(1+\tau_{1} s^{\alpha}\right)}{\left(1+\tau_{1} s^{\alpha}\right)\left(1+\tau_{2} s\right)}
$$

It can be rearranged to complex form by Euler formula $s^{\alpha}=\omega^{\alpha}[\cos (x)+j \cdot \sin (x)]$, where $x=\alpha \pi / 2$, as: 


$$
K_{C}(j \omega)=\frac{A+B+B \tau_{1} \omega^{\alpha} \cos (x)+j\left(B \tau_{1} \omega^{\alpha} \sin (x)+A \tau_{2} \omega\right)}{\left[\begin{array}{l}
1+\tau_{1} \omega^{\alpha} \cos (x)-\tau_{1} \tau_{2} \omega^{1+\alpha} \sin (x)+ \\
+j\left(\tau_{1} \omega^{\alpha} \sin (x)+\tau_{1} \tau_{2} \omega^{1+\alpha} \cos (x)+\tau_{2} \omega\right)
\end{array}\right]}
$$

The overall phase response can be calculated from (4) and (5) respectively as:

$$
\begin{aligned}
& \varphi(\omega)=\tan ^{-1}\left(\frac{A \tau_{2} \omega+B \tau_{1} \omega^{\alpha} \sin (x)}{A+B+B \tau_{1} \omega^{\alpha} \cos (x)}\right)- \\
& -\tan ^{-1}\left(\frac{\tau_{2} \omega+\tau_{1} \omega^{\alpha} \sin (x)+\tau_{1} \tau_{2} \omega^{1+\alpha} \cos (x)}{1+\tau_{1} \omega^{\alpha} \cos (x)-\tau_{1} \tau_{2} \omega^{1+\alpha} \sin (x)}\right)
\end{aligned}
$$

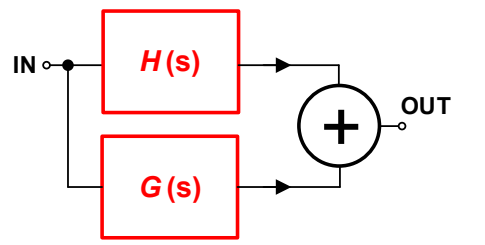

(a)

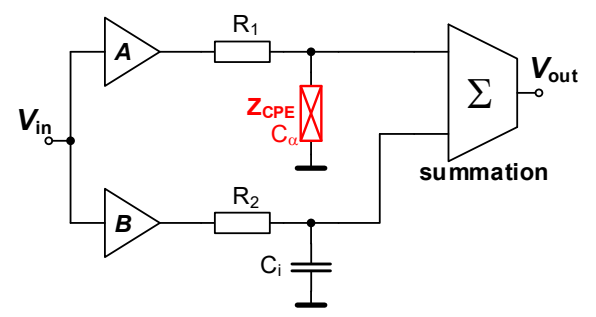

(b)

Fig. 1. The studied voltage-mode topology: a) general concept, b) the simplest circuit.

\section{STUDY OF BEHAVIOR}

These initial values of parameters were used: $\tau_{1}=\tau_{2}=50 \mu \mathrm{s}, \quad A=B=1$. It results into $R_{1}=R_{2}=1 \mathrm{k} \Omega, C_{\alpha}=50 \cdot 10^{-9} \mathrm{~F} / \mathrm{sec}^{1-\alpha}$ (for all tested values of $\alpha$ ) and $C_{\mathrm{i}}=50 \mathrm{nF}$. We are studying the proposed system in three aspects: a) variation of the pass band gain $(A=B, A=2 B$, $A=B / 2), \mathrm{b})$ variation of time constant $\left(\tau_{1}=\tau_{2}, \tau_{1}=2 \tau_{2}\right.$, $\left.\tau_{1}=\tau_{2} / 2\right)$, c) variation of the order of the fractional-order lowpass segment $(\alpha=1, \alpha=0.75, \alpha=0.5, \alpha=0.25)$. The Matlab and Mathcad environments are used for these mathematical analyses.

The resulting ideal behavior is documented by Fig. 2 Fig. 4. The values of constant parameters are included directly in figures. Variation of pass-band gains $A, B$ has direct impact on maximal available phase shift, pass-band and stop-band gain as well as slope changes between these bands. The resulting frequency responses of the structure in dependence on $A, B$ are shown in Fig. 2. Figure 3 indicates significantly lower impact of different ratios of time constants on resulting responses because of $A=B$. In comparison to the case with $A$ not equal to $B$ (Fig. 5 described below), the ratio of time constants should be in thousands in order to obtain any significant effect. Figure 4 shows results of intentional change of order $\alpha$. It has influence on stop-band behavior of the resulting low-pass response in this particular design. We used only parameter $B$ (pass-band gain of lossy integer-order integrator) for stepping from 0.1 up to 10 in order to see the full effect on overall behavior with particular setting of time constants: $\tau_{1}=50 \mathrm{~ms}, \tau_{2}=50 \mu \mathrm{s}, \alpha=0.25$, and $A=1$. Results in Fig. 5 indicates possibility of extension of area with maximal value of phase shift and forming of the shape (width of the lobe) by appropriate (increased) ratio of time constants. Large distance of values of time constants and increasing gain $B$ extends area with maximal phase shift. Specific configuration of parameters may lead to wider range of "constant" phase than shown in Fig. 2 to Fig. 4.

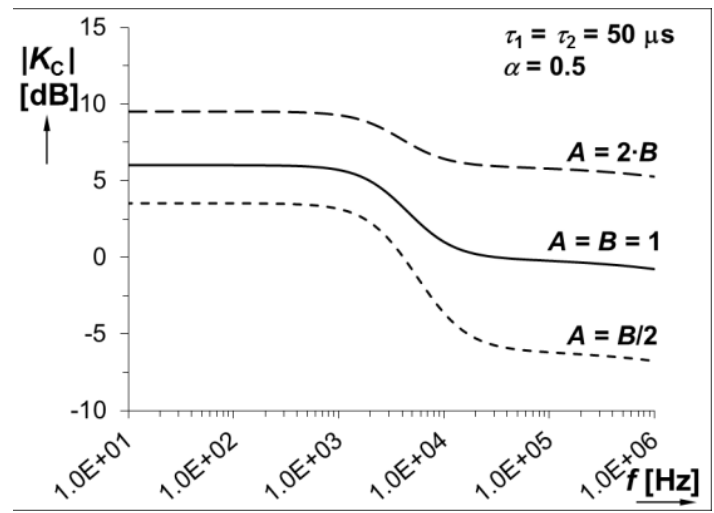

a)

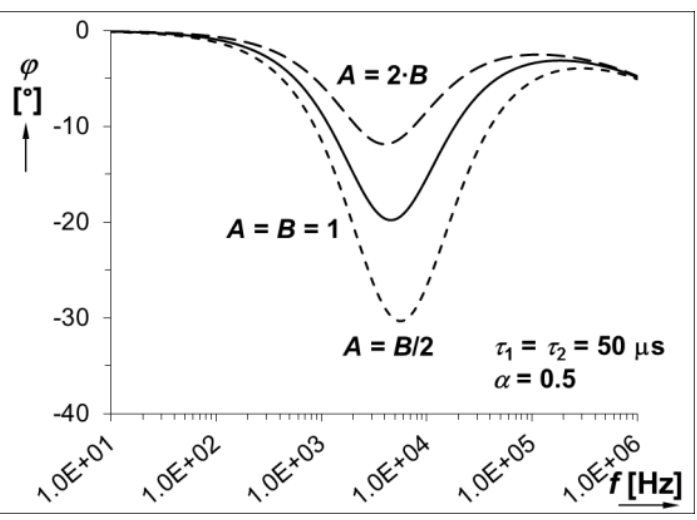

b)

Fig. 2. Ideal frequency responses of the topology for different ratios of passband gains $(A, B)$ : a) magnitude responses, b) phase responses.

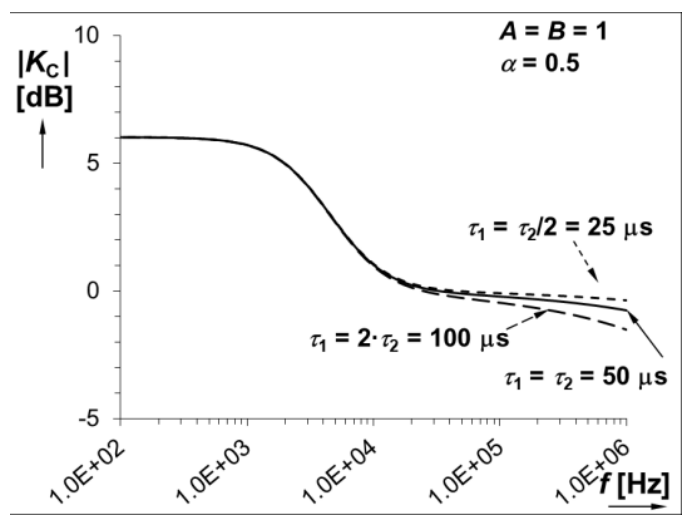

a) 


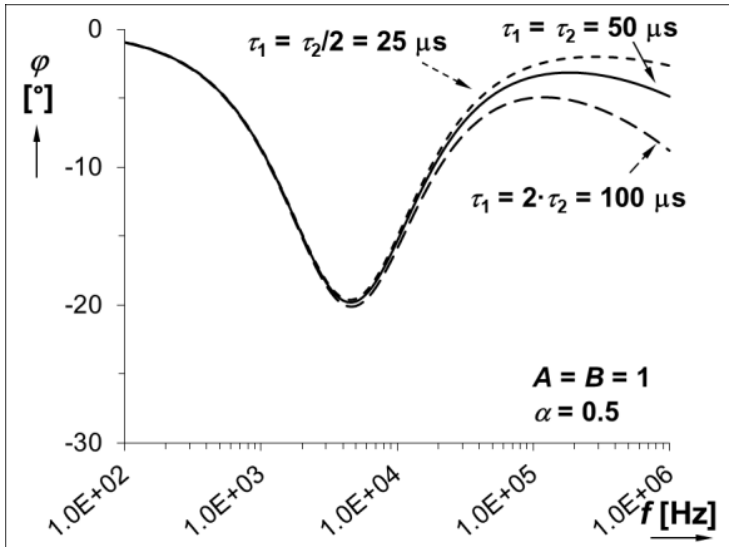

b)

Fig. 3. Ideal frequency responses of the topology for different ratios of time constants $\left(\tau_{1}, \tau_{2}\right)$ : a) magnitude responses, b) phase responses.

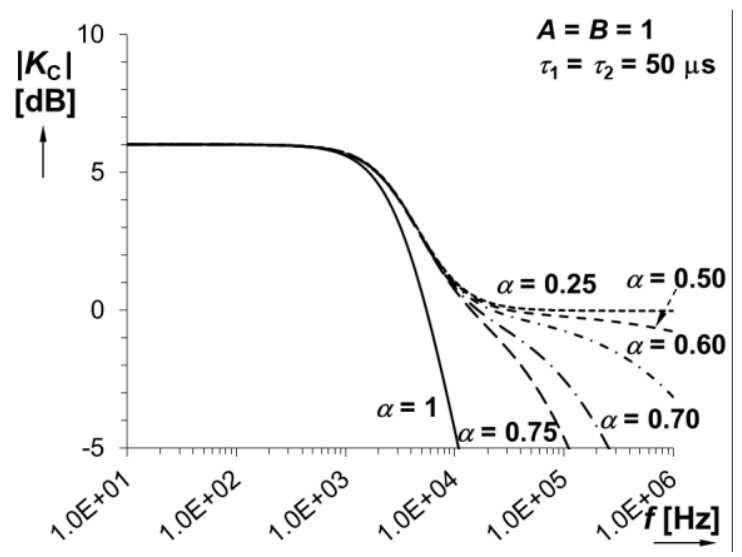

a)

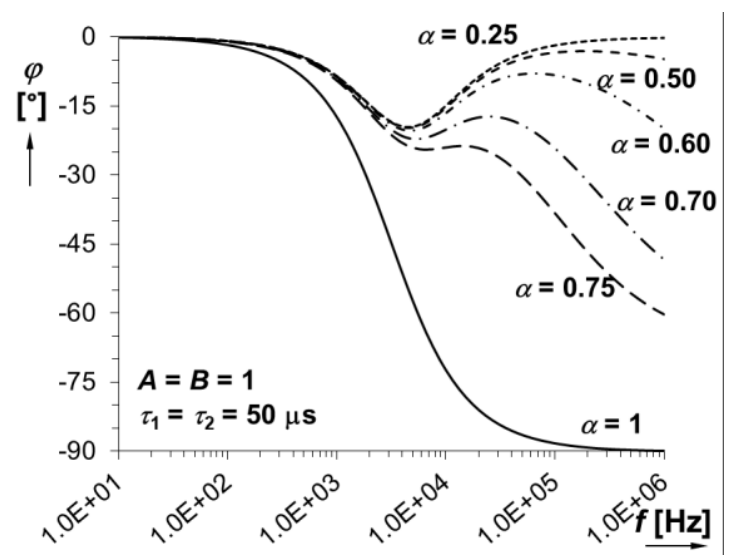

b)

Fig. 4. Ideal frequency responses of the topology for variation of order $\alpha$ : a) magnitude responses, b) phase responses.

\section{TESTS OF TOPOLOGY WITH APPROXIMANT OF FRACTIONAL- ORDER INTEGRATOR}

The magnitude and phase traces of approximated fractional-order part $H(\mathrm{~s})$ [23], used in the following verification, represent this setting of ideal model (2): $A=100$, $\tau_{1}=7 \mathrm{~s}, \alpha=0.25$ that fits the behavior of modified CPE in studied bandwidth inspired by [23]. Note that there is significant difference between approximated and ideal case at low frequencies due to limited validity of approximation for these frequencies. The integer order low-pass section $G(\mathrm{~s})$ has fixed time constant (3): $\tau_{2}=10 \mu \mathrm{s}$, that results into $R_{2}=1 \mathrm{k} \Omega$ and $C_{\mathrm{i}}=10 \mathrm{nF}$. The 3D plot (Fig. 6) with discussed ideal model indicates magnitude and phase changes for continuous variation of $H(\mathrm{~s})$ order in range $0 \leq \alpha \leq 1$.

Previous discussions considered ideal character of capacitor $C_{\alpha}$ used in fractional-order lossy block. We designed this CPE element by method shown in [16], [17] and analyzed system topology with PSpice macromodels of real off-the-shelf active elements. The full topology is shown in Fig. 7. The amplifier (VGA) is implemented by VCA 810 with controllable gain $\left(B=10^{2(\text { Vset_B-1) }}\right)$, the operational transconductance amplifier (OTA) uses well known OPA860 device, transconductance was fixed to $g_{\mathrm{m}}=1 \mathrm{mS}$ and differential difference amplifier (DDA) providing sum of two input voltages is based on AD8130. Each active element was supplied by $\pm 5 \mathrm{~V}$ voltage. Results of simulations of the topology with approximant of $C_{\alpha}$ and ideal element (phase constant in infinite range of frequencies) have been compared in 2D graph in Fig. 8 for three values of $B$. Note that the pass-band gain $B$ is variable by driving voltage $V_{\text {set_B. }}$. Figure 9 shows the CPE topology and all values of used elements.

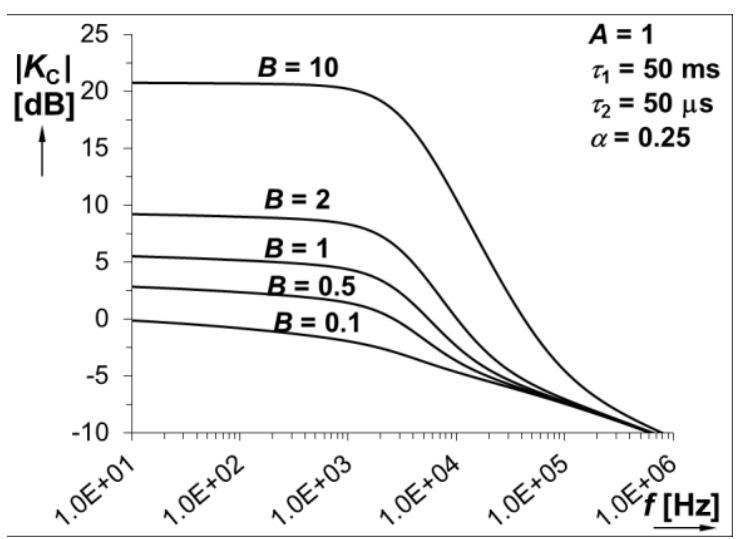

a)

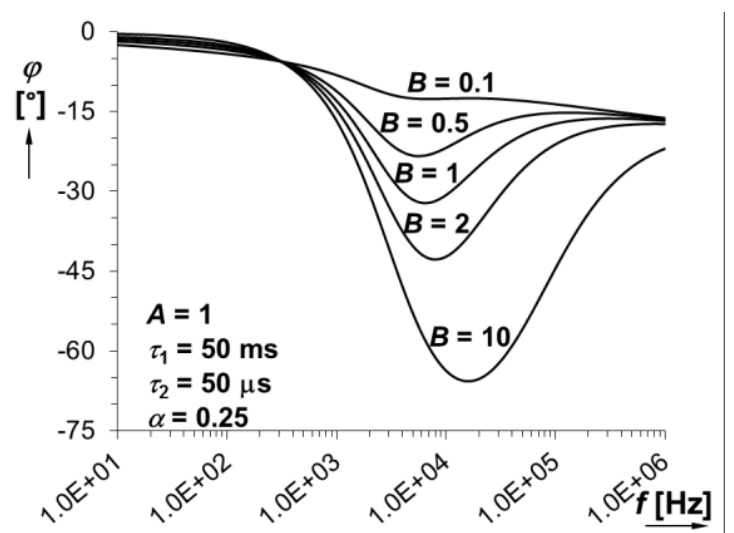

b)

Fig. 5. Ideal frequency responses of the topology for variation of gain $B$ : a) magnitude responses, b) phase responses. 


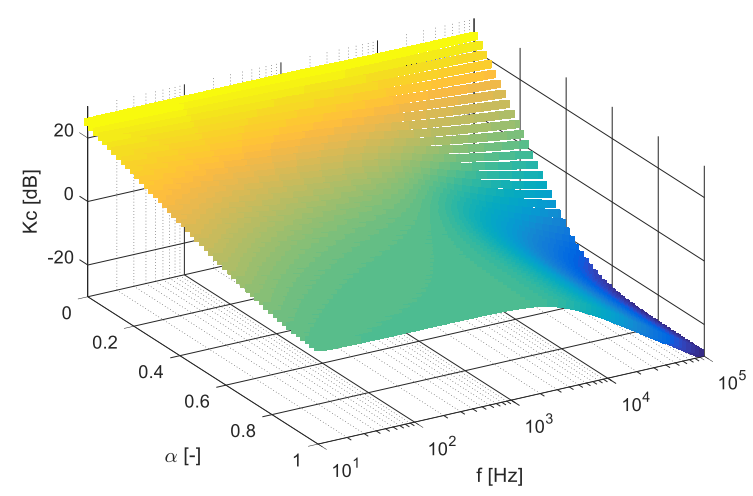

a)

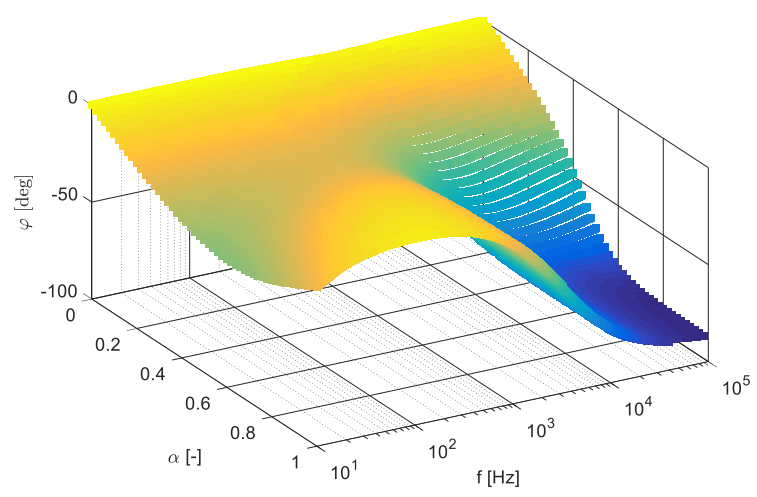

b)

Fig. 6. The 3D plot of studied topology for initial setting $\left(A=100, \tau_{1}=7\right.$, $B=1, \tau_{2}=10 \mu \mathrm{s}$ ) and variable $\alpha$ : a) magnitude vs order vs frequency dependence, $b$ ) phase vs order vs frequency dependence.

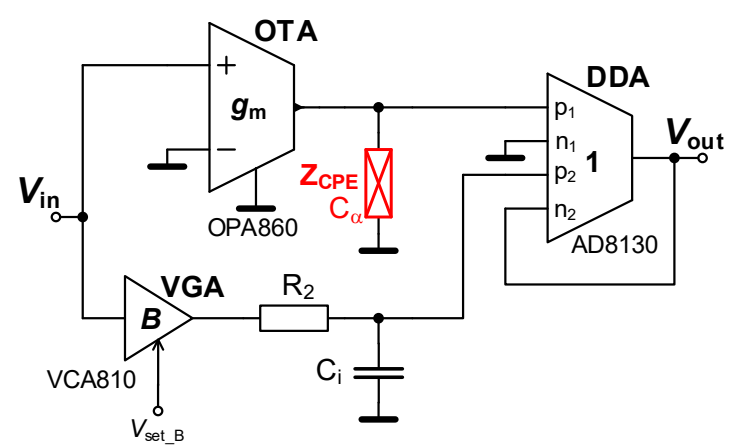

Fig. 7. Real implementation of topology from Fig. 1 tested by simulations.

It is clearly visible that some "constant" operational bandwidth (depending also on $B$ value) occurs approximately between $40 \mathrm{~Hz}$ and $1 \mathrm{kHz}$ (valid for all tested values of $B=0.1$, $1,10)$. The phase shift $\varphi$ reaches in this region $-5^{\circ}(B=10)$,
$-16^{\circ}(B=1)$ and $-23^{\circ}(B=0.1)$. The maximal phase ripple does not overcome $\pm 1.5^{\circ}\left(\varphi=-23^{\circ}\right)$ in this operational band. The theoretical phase shift $-22.5^{\circ}$ is maximal available phase shift for used CPE element (Fig. 9) in our fractional-order integrator. Therefore, increase of gain $B$ causes decrease of phase shift from maximal available value. It is clear that intentional additional pole of the low-pass section $G(\mathrm{~s})$ degrades operational bandwidth (it unintentionally influences zero in resulting function) of the $\mathrm{CPE}$ used in fractional-order part $H(\mathrm{~s})$ and, therefore, also significantly reduces useful bandwidth of the device and has impact on the phase ripple. Nevertheless, there are some effects worth further study (beyond of extend of this initial study). There is possibility to adjust (in limited bandwidth) the order (and phase shift) by single controllable parameter. The observed impact on trace slope in magnitude as well as shifting of "constant" phase in phase responses (in limited bandwidth) clearly documents and implies this hypothesis.

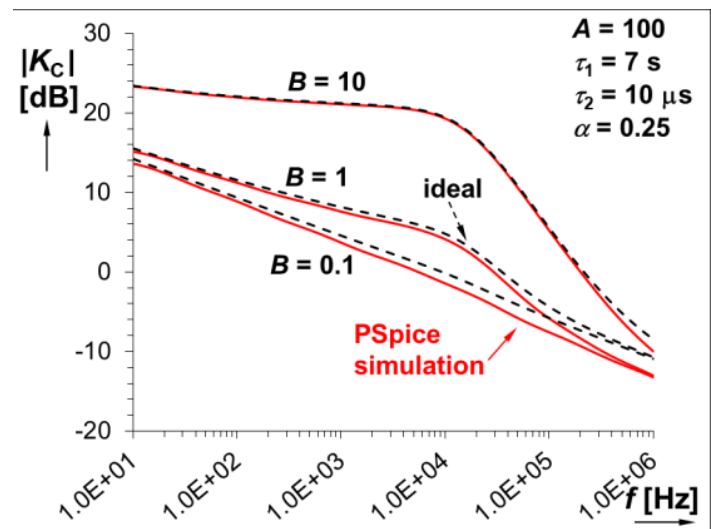

a)

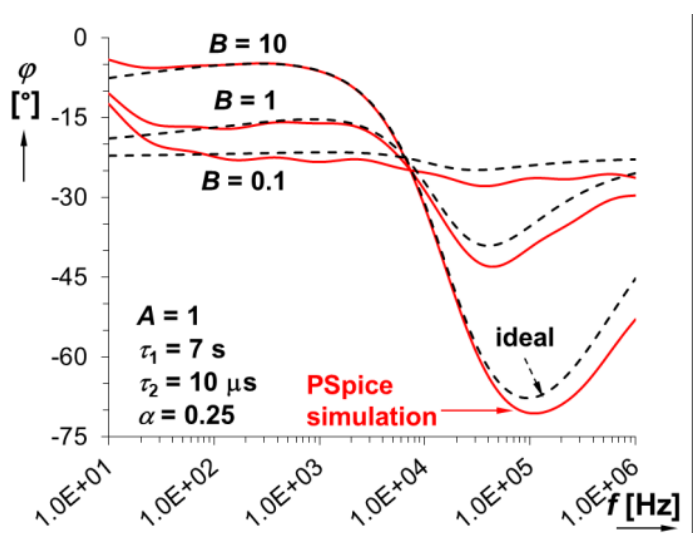

b)

Fig. 8. Frequency responses of the topology simulated in PSpice for variation of gain $B$ : a) magnitude responses, b) phase responses.

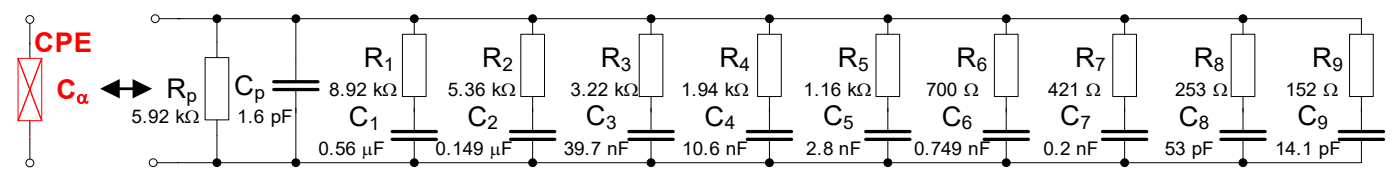

Fig. 9. RC chain of CPE (topology adopted from [23], values recalculated for this particular application). 


\section{CONCLUSION}

We presented system summing two different transfer functions. One of them is of integer order and second one is of fractional order, therefore, we can observe and study effects of interaction of them when control of parameters of their transfers is applied. Based on results of our initial study, some parameters, including variable time constants and especially pass-band gain of integer-order signal path, seems to be useful for shaping of the resulting response for various design requirements. It could be very useful because direct control of parameters of fractional-order response is usually much more complicated. Appropriate setting of time constants allowed us to verify variable value of "constant" phase in approximately one and half decade of operational bandwidth for three different phase shifts from $-23^{\circ}$ up to $-5^{\circ}$ when voltage pass-band gain $B$ was varied from 0.1 up to 10 . Bandwidth showed in these results is set from $10 \mathrm{~Hz}$ up to $1 \mathrm{MHz}$ because important details for our study belongs to this range or further change of responses (approaching of traces to $-22.5^{\circ}$ ) occurs at frequency higher than $1 \mathrm{THz}$ (after 6 decades). Of course simulation tools and models would be only theoretical in this band, therefore, frequency range is limited as mentioned above. The development of methodology requires further precise study and work on this topic including experimental verification.

\section{REFERENCES}

[1] A. S. Elwakil, "Fractional-order circuits and systems: An emerging interdisciplinary research area", IEEE Circuits and Systems Magazine, vol. 10, no. 4, pp. 40-50, 2010. DOI: 10.1109/MCAS.2010.938637

[2] A. G. Radwan, A. S. Elwakil, A. M. Soliman, "Fractional-order sinusoidal oscillators: design procedure and practical examples", IEEE Transactions on Circuits and Systems I: Regular Papers, vol. 55, no. 7, pp. 2051-2063. DOI: 10.1109/TCSI.2008.918196

[3] A. Kartci, N. Herencsar, J. Koton, L. Brancik, K. Vrba, G. Tsirimokou, C. Psychalinos, "Fractional-order oscillator design using unity-gain voltage buffers and OTAs", in Proceedings of the 60th International Midwest Symposium on Circuits and Systems (MWSCAS), 2017, pp. 555-558, DOI: 10.1109/MWSCAS.2017.8052983

[4] E. M. Hamed, A. M. AbdelAty, L. A. Said, A. G. Radwan, "Effect of Different Approximation Techniques on Fractional-Order KHN Filter Design", Circuits, Systems, and Signal Processing, vol. 37, no. 12, pp. 5222-5252, 2018. DOI: 10.1007/s00034-018-0833-5.

[5] D. Kubanek, T. Freeborn, J. Koton, "Fractional-order band-pass filter design using fractional-characteristic specimen functions", Microelectronics Journal, vol. 86, no. 4, pp. 77-86, 2019. DOI: 10.1016/j.mejo.2019.02.020

[6] C. Vastarouchas, C. Psychalinos, A. S. Elwakil, A. A. Al-Ali, "Novel two-measurements-only Cole-Cole bio-impedance parameters extraction technique", Measurement, vol. 131, no. 1, pp. 394-399, 2019. DOI: 10.1016/j.measurement.2018.09.008

[7] I. Dimeas, I. Petras, C. Psychalinos, "New analog implementation technique for fractional-order controlled: a de motor control", $A E U$ International Journal of Electronics and Communications, vol. 78, no. 8, pp. 192-200, 2017. DOI:10.1016/j.aeue.2017.03.010

[8] O. Domansky, R. Sotner, L. Langhammer, J. Jerabek, C. Psychalinos, G. Tsirimokou, "Practical Design of RC Approximants of Constant Phase Elements and Their Implementation in Fractional-Order PID Regulators Using CMOS Voltage Differencing Current Conveyors", Circuits, Systems and Signal Processing, online first, 2018, DOI: $10.1007 /$ s00034-018-0944-z
[9] L. Kadlcik, P. Horsky, "A CMOS Follower-Type Voltage Regulator With a Distributed-Element Fractional-Order Control", IEEE Transactions on Circuits and Systems I: Regular Papers, vol. 65, no. 9 , pp. 2753-2763, 2018. DOI: 10.1109/TCSI.2018.2808879

[10] G. Tsirimokou, C. Psychalinos, A. S. Elwakil, K. N. Salama, "Experimental behavior evaluation of series and parallel connected constant phase elements", AEU - International Journal of Electronics and Communications, vol. 74, no. 4, pp. 5-12, 2017. DOI: 10.1016/j.aeue.2017.01.010

[11] A. Kartci, A. Agambayev, N. Herencsar, K. N. Salama, "Series-, Parallel-, and Inter-Connection of Solid-State Arbitrary Fractional-Order Capacitors: Theoretical Study and Experimental Verification", IEEE Access, vol. 6, no. 1, pp. 10933-10943, 2018. DOI: 10.1109/ACCESS.2018.2809918

[12] M. Krishna, S. Das K. Biswas, B. Goswami, "Fabrication of a fractional order capacitor with desired specifications: a study on process identification and characterization, IEEE Trans Electron Devices, vol. 58, no. 11, pp. 4067-4073, 2011. DOI: 10.1109/TED.2011.2166763

[13] A. M. Elshurafa, M. N. Almadhoun, K. N. Salama, H. N. Alshareef, "Microscale electrostatic fractional capacitors using reduced graphene oxide percolated polymer composites", Applied Physics Letters, vol. 102 , no. 23, pp. 232901-232904, 2013. DOI: 10.1063/1.4809817.

[14] A. Adhikary, M. Khanra, S. Sen, K. Biswas, "Realization of carbon nanotube based electrochemical fractor", in Proceedings of IEEE International Symposium on Circuits and Systems (ISCAS), 2015, pp. 2329-2332. DOI: 10.1109/ISCAS.2015.7169150.

[15] P. Ushakov, A. Shadrin, D. Kubanek, J. Koton, "Passive fractionalorder components based on resistive-capacitive circuits with distributed parameters", in Proceedings of 39th International Conference on Telecommunications and Signal Processing (TSP), 2016, pp. 638-462. DOI: 10.1109/TSP.2016.7760960

[16] J. Valsa, P. Dvorak, M. Friedl, "Network model of the CPE", Radioengineering, vol. 20, no. 3, pp. 619-626, 2011.

[17] J. Valsa, J. Vlach, "RC models of a constant phase element", International Journal of Circuit Theory and Applications, vol. 41, no. 1, pp. 59-67, 2013. DOI: 10.1002/cta.785

[18] G. Tsirimokou, A. Kartci, J. Koton, N. Herencsar, C. Psychalinos, "Comparative Study of Discrete Component Realizations of FractionalOrder Capacitor and Inductor Active Emulators", Journal of Circuits Systems and Computers, vol. 27, no. 11, pp. 1850170-1-1850170-26, 2018. DOI: $10.1142 / \mathrm{S} 0218126618501700$

[19] S. Kapoulea, C. Psychalinos, A. S. Elwakil, A. G. Radwan, "Oneterminal electronically controlled fractional-order capacitor and inductor emulator", AEU - International Journal of Electronics and Communications, online first, 2019. DOI: 10.1016/j.aeue.2019.03.002

[20] R. Sotner, J. Jerabek, N. Herencsar, L. Langhammer, J. Petrzela, T. Dostal, "Methods for Extension of Tunability Range in Synthetic Inductance Simulators", Eletronika ir Elektrotechnika, vol. 24, No. 3, pp. 41-45, 2018. DOI: $10.5755 /$ j01.eie.24.3.20976

[21] S. Kapoulea, C. Psychalinos, A. S. Elwakil, "Single active element implementation of fractional-order differentiators and integrators", $A E U$ - International Journal of Electronics and Communications, vol. 97, no 12, pp. 6-15, 2018. DOI: 10.1016/j.aeue.2018.09.046

[22] R. Bertsias, C. Psychalinos, A. S. Elwakil, L. Safari, S. Minaei, "Design and application examples of CMOS fractional-order differentiators and integrators", Microelectronics Journal, vol. 83, no. 1, pp. 155-167, 2019. DOI: 10.1016/j.mejo.2018.11.013

[23] R. Sotner, J. Jerabek, O. Domansky, N. Herencsar, A. Kartci, J. Dvorak, "Practical Design of Fractional-Order Oscillator Employing Simple Resonator and Negative Resistor", in Proc. of the 10th International Congress on Ultra Modern Telecommunications and Control Systems and Workshops (ICUMT), 2018, pp. 248-251. DOI: 10.1109/ICUMT.2018.8631226 